\title{
The Typing Test System Based on Network
}

\author{
Yaohui Li ${ }^{1}$, Meijin Yuan ${ }^{2}$,Jianxiong Wang ${ }^{1}$, Lili Zhou ${ }^{1}$, Kejian Yang ${ }^{1}$ \\ 1. Department of Computer \\ Hebei Institute of Architecture \& Civil Engineering \\ Zhangjiakou City, Hebei Province, China \\ E-mail: lyhfirst@163.com \\ 2. Nursing Department \\ First Affiliated Hospital of Hebei North University \\ Zhangjiakou City, Hebei Province, China
}

\begin{abstract}
A new idea about realizing the typing exam system was proposed. The exam system is based on network. It takes full advantage of the Browser/Server model, such as the strong interaction, high rapidity, security and the strong share. The exam system is developed with ASP. It is simple to install, easy to use and full-featured. Through the practical application, it is found that the exam system is satisfied the requirements of typing exam. There is not report of similar software up to now.
\end{abstract}

Keywords- Typing Exam; Network; ASP

\section{INTRODUCTION}

The computer is widely used. The operation and use of any application software are inseparable from the text entry, so the text entry operation is the foundation of computer management [1]. We should master the basic operation and application, so that we can better use the computer. There are a lot of popular typing exam softwares, but the main purpose of these developers is to allow users to practice and self-testing. If these softwares is used to the examinations for school typing class, or used as a typist examination, a lot of problems would be showed [2]. Most of the typing exam software is standalone version. If this type of software is used for typing exam, the software installation would spend a lot of time and trouble to view and record results to each computer [3]. Although a small number of online typing exam software, but they are based on Client/Server model, it is still very time-consuming to install.

B/S model (Browser/Server model, Browser/Server structure) is a network model [4]. WEB browser is the most important applications of the client. This model unifies the client and the core of system function to the server, so the system development, maintenance and use is simplified. The biggest advantages of $\mathrm{B} / \mathrm{S}$ model is that you can operate at anywhere without having to install any special software, as long as there is a computer with Internet access. The client side has zero maintenance. Expansion of the system is very easy. Web service is the latest technology trends to construct a distributed, modular application [5]. So we decided to develop the network typing exam system based on $\mathrm{B} / \mathrm{S}$ model.

\section{SYSTEM ANALYSIS}

\section{A. User Analysis}

The exam managers and the examinees are user of the exam system. This is a software for examination and usually students do not have access to the exam system. The interface should be as simple as possible so that the examinees can get started without training. For the exam manager, they call for a system that is full-featured enough and easy to use.

\section{B. Software Analysis}

Currently, most of the computer rooms are equipped with a protection card. Installing the software for each exam takes a great amount of work. Because of use B/S model, the server requirements windows operating system and IIS. The computer for examinee will only requirements WEB browser. The exam can start after the exam system is installed and simply configured on the server.

\section{Hardware Analysis.}

The computer room is a networked computer configuration. This configuration is enough for this exam system. Developers need not to consider the machine speed, memory and other issues.

\section{Technical Analysis}

The exam system is designed and developed based on ASP. Microsoft Active Server Pages (ASP) is a server-side scripting environment that can be used to create dynamic, interactive and efficient Web server applications. An ASP page is an HTML page that contains server-side scripts that are processed by the Web server before being sent to the user's browser. You can combine ASP with Extensible Markup Language (XML), Component Object Model (COM), and Hypertext Markup Language (HTML) to create powerful interactive Web sites.

Server-side scripts run when a browser requests an .asp file from the Web server. ASP is called by the Web server, which processes the requested file from top to bottom and executes any script commands. It then formats a standard Web page and sends it to the browser. 


\section{SYSTEM DESIGN AND IMPLEMENTATION}

\section{A. Primary Function}

Server-side function include: (1) Set the examination time limit. (2) Set the exam text. (3) Check in the data of the examinee. (4) Start the examination. (5) Record information of examinees login. (6)Receive data from each exam computer and save it. (7) Calculate and save the results. (8) Query examinee information.

Exam-side function include: (1) Login system. (2) The countdown to the end in accordance with the examination time limit. (3) Select randomly and display the text that examinee should input, wait for examinees to input text. (4) Display typing speed, remaining time and the number of words that examinee input incorrectly. (5) At end of the examination, the exam system can automatically carry out an assignment. (6) Show exam score.

\section{B. Description of the Examination Process}

1) Set the examination time, specify the exam text and confirm the start on server.

2) Examinee login system.

3) The examinees can input the text into the exam interface.

4) After input all text, examinee can carry out an assignment. Arrived at the end time, the exam system can carry automatically out an assignment.

5) Process examination results on server-side, the examination ended.

\section{Design Process}

1) The exam managers can specify one or more exam text article. If a exam text specified, all the examinees must enter exactly the same content. If multiple articles specified, the exam system will randomly select a text, so that the contents between of the adjacent examinees should input is inconsistent. Exam text and exam time should be based on the length, difficulty and exam standards.

2) In order to enter the exam system, examinee should input the ticket number and name. If the input is not correct, there will be tips to help examinee to change. If the ticket number and the name that examinee inputted has been logged, he will be prompted to enter a second password. The second password controlled by the invigilator teachers. In order for examinee familiar with the interface, the system set up a practice number that is unlimited by a second password.

3) After logging into the exam system, the interface can be seen. At the top of screen the exam time, remaining time, the number of words examinee inputted, the number of wrong words examinee inputted, examinee name is displayed. Examinee may input text in the below input box. At begin of the design, two input boxes are shown. One displays the examination text content and the other accept the text that examinee inputted. During the test of this system, it is found that examinee is not easy to locate the input text in progress and the wrong words. Therefore the system is improved. The examination content is displayed in a div field. The text examinee input displays with a textarea, a text input box whose type is set to hidden save the exam text. After examinee input a character, compared the value of input box with the value of textarea, a string is generated. Add the HTML tags that control color of character into the string, so that the correct words that examinee input is blue and the wrong words that examinee input is red. Update the value of div with this string. This makes it easy to identify the wrong words and the progress.

Exam again and it is found that the scroll bar of textarea and the scroll bar of div had to adjust often in order to the text that display in textarea and in div field is same. Therefore the roll function is designed, which make the scroll bar of div associated to the scroll bar of textarea. When the scroll bar of textarea scrolling up and down, div scroll bar automatically scroll accordingly.

In all the typing exam system based on B/S model, there is not report similar to the above design.

4) In order to prevent examinee cheating in the examination process, the exam system prohibit the use of copy, paste, and the functions of mouse's right button.

5) The time is recorded by the server and the examinee's computer. The time recorded by the examinee's computer is used for the automatic control of the end. The time recorded by the server is used for the score calculation.

6) The results of the examinees calculated by the number of wrong words, the number words of exam text, examinees enter the number of words, and the time the examinee spent.

\section{CONCLUSION}

The system has been used for the actual exam. The exam system is installed on the server and configured. It is easy to install. A lot of time and effort is saved. The functions of the system are run correctly. Examinees can quickly become familiar with the system. The system can be used for typing exam and also be used in exercises. There is not report of similar software up to now.

\section{ACKNOWLEDGMENT}

This work was supported by a grant from the Science Technology Research and development Guidance program of Zhangjiakou City (No. 060152) and Technology Research and Development Guidance Program of Hebei Province (No. 052135125)

\section{REFERENCES}

[1] Zhengkui Zhong, Ruiping Liao, "Design and Implementation of Typing Exam Software Based on VB,” Science \& Technology Information,, 2009, (30) : 201 - 202.

[2] Deqiang Liu, "Design of students Typing Competitive Software" Journal of Wuxi Institute of Technology, 2006,(2), 24-25

[3] Youyi Cui, "Design and Implementation of Typing Exam Software on LAN,”. Heilongjiang Science \& Technology Information, 2010, (12):66-67

[4] Jiangwen Zhang, Hong Rao, Sujuan $\mathrm{Hu}$, "Design of Online Examination System Based on B / S Model,” Computer Knowledge and Technology,2009,(4)56-58

[5] Jin Zhang, Shihuan Ma, "Realize the Network Typing Competition System Based on Web Services," Science \& Technology Information, 2009,(35):478-479. 